\title{
Incomplete Superficial Palmar Arch - Clinical Significance
}

\author{
(Dr.Challa. Ratna Prabha ${ }^{1}$, Dr.BvindalaBhagalakshmi ${ }^{2}$, \\ Dr.NamburiBhanuSudhaParimala ${ }^{3}$, Dr.ChandakaSwathiPoornima ${ }^{4)}$ \\ (ASSOCIATE PROFESSOR ${ }^{l}$, Department of Anatomy, Dr. PSIMS \& RF, Chinoutpalli, Gannavaram, Krishna \\ $D t, A P$, India)
}

(PROFESSOR\& HOD²,Department of Anatomy, Dr. PSIMS \& RF, Chinoutpalli, Gannavaram, Krishna Dt, AP, India)

(ASSISTANT PROFESSOR ${ }^{3 \& 4}$, Department of Anatomy, Dr. PSIMS \& RF, Chinoutpalli, Gannavaram, Krishna Dt, AP, India)

\begin{abstract}
The hands receive profuse blood supply by superficial and deep palmar arches (SPA \& DPA), though both arches are constituted by radial and ulnar arteries, variations are constantly observed in SPA.

During routine dissections we observed unilateral incomplete SPA being formed by superficial branches of radial and ulnar arteries in the right hand of an old male cadaver. These two arteries remained independent without anastomosis forming incomplete arch. The superficial branch of radial artery supplied the thumb and radial side of index finger by its two branches - the arteriaprincepspollicis and arteriaradialisindicis. Normally these two branches are given by radial artery but here they are given by a branch of radial artery.

The presence of an incomplete SPA as in this case is a potential danger in RA harvesting for CABG. Variations in the SPA play a key role in microvascular surgical procedures of hand, RA interventions and arterial graft applications.
\end{abstract}

Key Words: Superficial palmar arch, deep palmar arch, the Arteriaprincepspollicis, Arteriaradialisindicis, Coronary artery bypass graft, Radial artery.

\section{Introduction}

The functional importance of the hand is revealed by its profuse blood supply by means of two arterial arcades in the palm, the superficial and deep palmar arches that are located superficial and deep to the flexor tendons and lumbrical muscles. Though the SPA and DPA are contributed by the radial and ulnar arteries, variations in the SPA are innumerable. Hence the SPA remained as a star attraction for the surgeons and anatomists as it plays a pivotal role in arterial graft applications, reconstructive hand surgeries, radial artery cannulations and radial artery forearm flap. A classic type of SPA is formed by the anastomosis between superficial branch of radial and ulnar arteries [1]. About $1 / 3^{\text {rd }}$ of SPA formation is by ulnar artery alone and another $1 / 3^{\text {rd }}$ by anastomosis between ulnar artery and superficial branch of radial artery and remaining $1 / 3^{\text {rd }}$ of arch is completed by ARI or APP or median artery [2].

From the convexity of the arch one proper palmar digital branch and three common palmar digital branches arise. The proper palmar digital branch is for the medial side of little finger. Each common digital branch divides in to two proper digital branches and supplies contiguous sides of medial four fingers. The radial side of index finger and the thumb are supplied by the ARI and APP which are the branches of radial artery [3].

The variations in the above pattern of formation are many. The knowledge of normal and abnormal patterns of SPA is an essential prerequisite for all hand surgeons before attempting surgical procedures. Modern medical technology demands a comprehensive but adequate knowledge of variations from surgeons in surgical procedures such as arterial cannulations, radial forearm flap, radial artery harvesting for CABG etc. Gellman classified the SPA as complete and incomplete types [4]. In the complete SPA there will be an anastomosis between formative vessels. In the incomplete SPA there will be absence of anastomosis in the formative vessels. This classification is simple and currently in use.

Coleman \& Anson subdivided the complete SPA into five varieties [5].

Type A: Classic type - Presence of anastomosis between superficial branches of ulnar and radial arteries

Type B: SPA is formed by ulnar artery alone.

Type C: Arch formed by median and ulnar arteries

Type D: Radial - median - ulnar arch

Type E: Arch formed by ulnar artery and vessels from the deep arch

Coleman \& Anson subdivided the incomplete SPA in to four varieties [5].

Type A: There is no anastomosis between superficial branches of radial and ulnar arteries 
Type B: SPA formed by ulnar artery alone

Type C: SPA formed by ulnar and median arteries without anastomosis

Type D: SPA formed by ulnar, median and radial arteries without anastomosis

In the present case we observed an incomplete arch that belongs to incomplete SPA - type A of Coleman \& Anson's classification.

\section{Case Report}

During routine dissections for the undergraduate students we found an incomplete SPA in the right hand and classic type of SPA in the left hand of an old male cadaver.

This incomplete SPA was constituted by superficial branch of radial artery and superficial branch of ulnar artery which entered the palm passing superficial to the flexor retinaculum. After providing a deep branch it took a gentle curve towards laterally giving a proper palmar digital artery (PPDA) to the medial side of little finger and three common palmar digital arteries (CPDA) which proceed to the interdigital clefts where they bifurcate and supply the contiguous sides of little - ring, ring - middle and middle - index fingers respectively.

The superficial branch of radial artery entered the palm crossing the thenar eminence and reaches the $1^{\text {st }}$ web space. Here it bifurcates to supply the thumb and radial side of index finger through the APP and ARI respectively. Normally the APP and ARI are the branches given by radial artery. There is no anastomosis between superficial branch of ulnar artery and superficial branch of radial artery. Hence the arch is incomplete and it is a potential danger in radial artery harvesting for $\mathrm{CABG}$ and also in complicated surgical procedures when the hands are injured. In this case the APP and ARI which are very important branches to the thumb and index finger are given by superficial branch of radial artery.

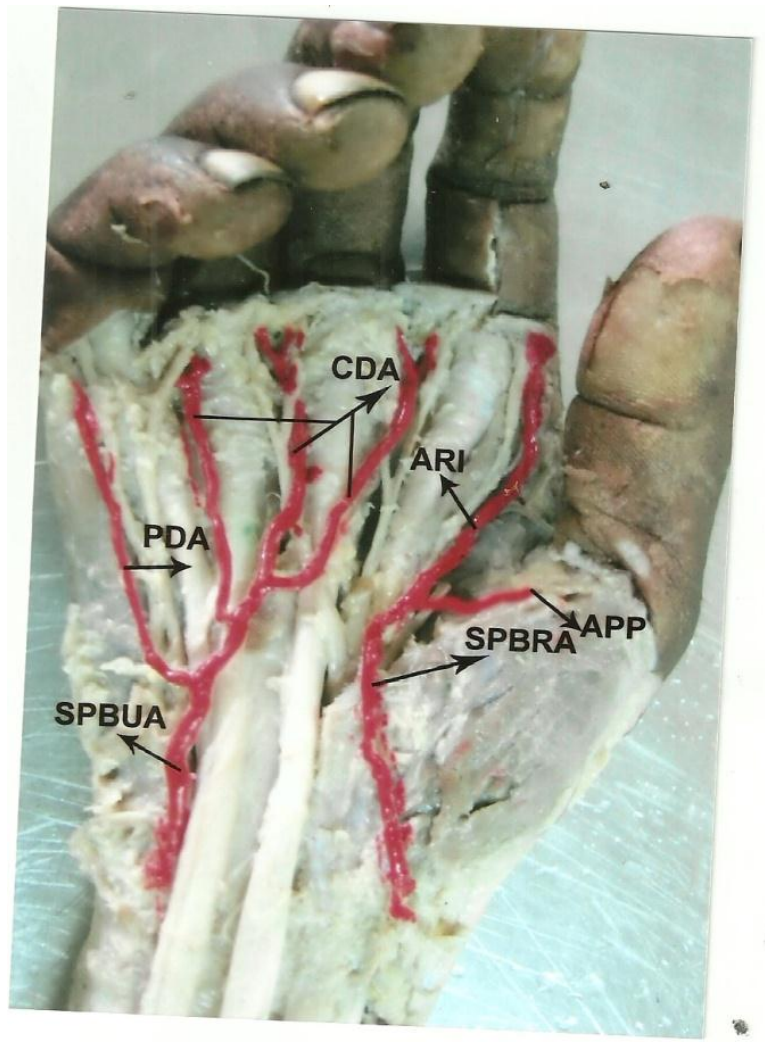

\section{LEGENDS:}

SPA - Superficial palmar arch, DPA - Deep palmar arch, APP - Arterial princepspollicis, ARI Arteriaradialisindicis, CABG - Coronary artery bypass graft, RA - Radial artery, CDA - Common digital artery, PDA - Proper digital artery, SPBRA - Superficial palmar branch of radial artery, SPBUA - Superficial palmar branch of ulnar artery

\section{Discussion}

The importance of variations in SPA is highlighted since from the time of harvesting radial artery as arterial graft for CABG. Complete SPA was observed by Coleman \& Anson in $78.5 \%$ cases, Loukas, Holdman and Holdman [6] in $90 \%$ cases. Ikeda et al [7] in $96.4 \%$ cases, Kalsey and Singla [8] in $78 \%$ cases, Al - turkand 
Metcalf [9] in $84 \%$ cases and Jelicic et al [10] in $55 \%$ cases. The incomplete SPA was reported by Loukas, Holdman and Holdmanin $10 \%$ cases, Kalsey, Patnaik and Singla in $16 \%$ cases, Al - turk and Met calf in 21.47 $\%$ cases, Coleman \& Anson in $21.5 \%$ cases and Gellman et al in $84.4 \%$ cases.

The present case is an incomplete SPA formed by superficial branches of radial and ulnar arteries without anastomosis belongs to incomplete SPA type A. This type A incomplete SPA was reported by Coleman \& Anson in $3.6 \%$ cases, Olave et al in $15 \%$ cases and Arjuo et al in $13.33 \%$ cases. Usually ulnar artery is constant in the SPA and the cause of variation is always radial artery [11]. Bergman, Thomson, Afifi et al (1988) [12] stated that according to Jaschtschinski if SPA formation is absent, the superficial branch of radial artery supplies the lateral fingers. In those hands RA harvesting for CABG results in ischemia and gangrene of the fingers. In the present case of incomplete SPA superficial branch of radial artery divides into two branches as APP and ARI to supply the thumb and radial side of index finger instead of direct branches from the radial artery. So in this case RA harvesting leads to ischemia and gangrene of fingers. The clamping of RA is contraindicated in cases of absence of collateral flow through ulnar artery as it causes ischemia and gangrene of fingers [13]. In the present case as there is no collateral flow through ulnar artery, clamping of RA is deleterious. The patients should be screened before harvesting the RA to confirm the presence of viable collateral circulation in the hand.

To assess the exact arterial pattern and collateral circulation, investigations like modified Allen's test, Doppler ultra sound, arterial angiography and pule oximetry are to be done routinely before any surgical procedures on hand [14]. The Doppler and angiography show the arterial pattern and the Allen's test can be used as a bed side evaluation to assess the collateral circulation.

A comprehensive knowledge of gross variations of SPA is a prerequisite for all surgeons before handling surgical procedures like arterial repairs, RA harvesting and RA interventions for the safe and successful outcome.

\section{Conclusion}

In the present days the best choice for CABG is the radial artery. In cases where SPA is classic or complete, RA can be harvested without any post operative digital ischemic changes leading to gangrene [14]. But in cases like our present case where there is no collateral circulation between the formative vessels, the radial artery cannot be harvested but if done the lateral side of the hand shows ischemic changes leading to gangrene of the fingers. This is a small contribution to the huge knowledge already existing.

\section{References}

[1]. Fazanvps B C T, Da silva JH, Caetano AG, Filho OAR. Superficial palmar arch: An arterial diameter study. J Anat 2004, 204:30711.

[2]. Johnson D, Ellis H, Collins P. Wrist and hand. Standring S, Ellis H, Healy Jc, Johnson D, Williams A, Grays Anatomy. 39 ${ }^{\text {th }}$ Ed., Edinburgh, Churchill Livingstone. 2005; 929 - 930.

[3]. Datta AK. Essentials of Human Anatomy. Superior and inferior extremities. $2^{\text {nd }}$ Ed., Calcutta, Current books international. $2000 ; 99$ $-100$

[4]. Gellman H, Botte MJ, Shankwiler J, Gelberman RH. Arterial pattern of the deep and superficial palmar arches. ClinOrthopRelat Res. 2001; 383:41 - 46 .

[5]. Coleman SS, Anson BJ. Arterial patterns in the hand based upon the study of 650 specimens. SurgGyyyynecol Obstet. 1961; 113: $409-424$.

[6]. Loukas, M, Holdman, D. And Holdman, S. Anatomical variations of the superficial and deep palmar arches. Folia Morphologica (Warss), 2005; 64(2): $78-83$.

[7]. Ikeda a, Ugawa A, Kazihara Y, Hamada N. Arterial patterns in the hand based on three dimensional analysis of 220 cadaver hands. J Hand Surg 1988; 13:501 - 509.

[8]. Kalsey and Singla, RK. Palmar arterial arches. A morphological study. Journal of Anatomical Society of India, 2002, 51(2):187 193.

[9]. AI - Turk M, Metcalf WK. A study of the superficial palmar arteries using the Doppler Ultrasonic Flowmeter. Journal of Anatomy, 1984, 138 (1): $27-32$

[10]. Jelicic N, Gajisin S, Zbrodowski A. Arcus Palmaris superficialis, ActaAnat (Basel). 1988; $132: 187$ - 190.

[11]. Ottone NE, Prum N, Dominguez M, Blast E, Medan C, Schinzato S, Finkelstein D. And Bertone VH. Analysis and clinical importance of arterial palmar irrigation and its variants over86 cases. International journal of Morphology, $2010 ; 28$ (1): 157 - 164.

[12]. Bergman RA, Thompson Sa, Afifi AK. And Saadeh FA. Compendium of human anatomical variations. Munich: Urban and Schwarzenberg, 1988:74-75.

[13]. Cambron BA, Ferrada P, Walcott R, Karthik S Kaynar AM. Demonstration of unilateral absence of the palmar arch without collateral circulation. 2006; 113: 6 - 7. Mid : 16391162

[14]. Batainch ZM, Moqattash. A complex variation on superficial palmar arch.FoliaMorphol.2006; 65: 406 - 409. 